\title{
Space-oriented segmentation and 3-dimensional source reconstruction of ictal EEG patterns
}

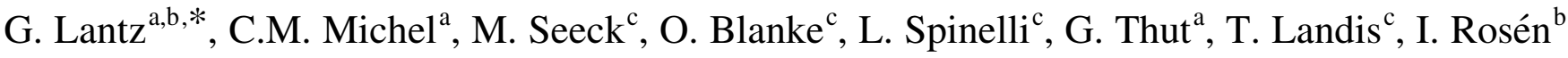 \\ ${ }^{a}$ Plurifaculty Program of Cognitive Neuroscience, Department of Neurology, University Hospital of Geneva, Geneva, Switzerland \\ ${ }^{\mathrm{b}}$ Department of Clinical Neurophysiology, Lund University Hospital, Lund, Sweden \\ ${ }^{\mathrm{C}}$ Laboratory of Presurgical Epilepsy Evaluation, Fondation Vaud-Genève, University Hospital of Geneva, Geneva, Switzerland
}

Accepted 8 January 2001

\begin{abstract}
Objectives: Characterization of the EEG pattern during the early phase of a seizure is crucial for identifying the epileptic focus. The purpose of the present investigation was to evaluate a method that divides ictal EEG activity into segments of relatively constant surface voltage distribution, and to provide a 3-dimensional localization of the activity during the different segments.

Methods: For each timepoint the electrical voltage distribution on the scalp (the voltage map) was determined from the digitized EEG recording. Through a spatial cluster analysis time sequences where the maps did not change much (segments) were identified, and a 3dimensional source reconstruction of the activity corresponding to the different mean maps was performed using a distributed linear inverse solution algorithm.

Results: Segments dominating early in seizure development were identified, and source reconstruction of the EEG activity corresponding to the maps of these segments yielded results which were consistent with the results from invasive recordings. In some cases a sequence of consecutive segments was obtained, which might reflect ictal propagation.

Conclusions: Segmentation of ictal EEG with subsequent 3-dimensional source reconstruction is a useful method to non-invasively determine the initiation and perhaps also the spread of epileptiform activity in patients with epileptic seizures. (C) 2001 Elsevier Science Ireland Ltd. All rights reserved.
\end{abstract}

Keywords: Epilepsy; Seizures; EEG; Temporal segmentation; Source reconstruction

\section{Introduction}

EEG recorded with surface electrodes plays an important role in the presurgical evaluation of patients with drug-resistant epileptic seizures. Although interictal epileptiform activity may give useful information, it has been shown that the interictal spike focus does not always coincide with the seizure onset area (So et al., 1989; Alarcon et al., 1994). For this reason, ictal EEG patterns are important for the localization and characterization of the epileptic focus in these patients (Risinger et al., 1989; Spencer et al., 1992; Brekelmans et al., 1995; Gotman et al., 1995; Ebersole and Pacia, 1996; Pacia and Ebersole, 1997; Kanemoto et al., 1997).

Different methods to quantitatively determine the origin and spread of epileptiform EEG activity during extra- or

\footnotetext{
* Corresponding author. Functional Brain Mapping Laboratory, Department of Neurology, University Hospital, 24 Rue Micheli-du-Crest, $\mathrm{CH}-$ 1211 Geneva, Switzerland. Tel.: +41-22-3728339; fax: +41-22-3728333.

E-mail address: lantz@cmu.unige.ch (G. Lantz).
}

intracranially recorded epileptic seizures have recently been introduced. Autoregressive modeling (Gath et al., 1992; Franaszczuk et al., 1994), time-frequency analysis (Franaszczuk et al., 1998; Osorio et al., 1998), wavelet analysis (for a review see Unser and Aldroubi, 1996), and methods related to non-linear dynamics (Pijn et al., 1991; Bullmore et al., 1994; Lehnertz and Elger, 1995) have been used for the analysis of seizure EEG in intracranial, surface, or combined intracranial and surface recordings.

One technique, Fourier spectral analysis, is the basis of the Fast Fourier Transform (FFT) dipole approximation method (Lehmann and Michel, 1990), which we have used in recent investigations to analyze surface recorded epileptic seizures of mesiotemporal origin (Lantz et al., 1999; Blanke et al., 2000). Several extracranially recorded seizures were analyzed in patients suffering from epilepsy of mesiotemporal origin. The full scalp frequency spectrum was determined for different periods during the seizures, power peaks in the spectra were identified, and source localization was performed for the frequencies corresponding to 
these peaks. In one of the investigations (Blanke et al., 2000), the temporal evolution of dominant peaks was also determined. One drawback with this approach, as with many of the other approaches mentioned above, is that the analysis requires a certain minimal analyzing period (around $2 \mathrm{~s}$ with a 128/s sampling rate), which limits the temporal resolution.

An alternative to the frequency analysis of the EEG is the determination of characteristic EEG patterns at seizure initiation in the time domain. One such technique is spatiotemporal dipole modeling (Scherg, 1990), which has been used to localize surface recorded ictal rhythms in temporal lobe patients with sublobar accuracy (Boon et al., 1996; Assaf and Ebersole, 1997). Other authors attempted to objectively characterize different segments of EEG patterns (Michael and Houchin, 1979; Sanderson et al., 1980; Lopes da Silva and Mars, 1987; Gath et al., 1983, 1991). The algorithms they proposed are based on the observation that periods of relatively stationary activity can be seen in the EEG recordings and that fairly abrupt changes occur from one stable segment to the other. Because of this relative stationarity and stepwise change of the signal, adaptive segmentation procedures can be used to detect these segment boundaries for a given recording channel. Such segmentation algorithms have been used in epilepsy by several authors in order to classify initiation and spread of the seizures (Gath et al., 1992; Wendling et al., 1997; Wu and Gotman, 1998), and it has been demonstrated that segmentation algorithms can be used not only to detect seizures but also to determine whether or not seizures are of the same type in a given patient.

While the methods described above used adaptive segmentation procedures on single channel data, we here propose a procedure that is based on the stability and changes of the whole scalp voltage topography over time. The method is based on the observation that the segment maps of consecutive sample points of spontaneous EEG remain stable for up to a few hundred milliseconds, and then promptly swift into another stable configuration. It has been suggested that these periods of relative stability manifest different brain functional states, and the term 'functional microstates' has been introduced (for a review see Lehmann et al., 1987). This interpretation is based on the fact that different electric field topographies on the scalp must be due to different configurations of the active neurons in the brain.

Some authors have used the microstate concept to quantitatively determine how EEG topography changes under different physiological and pathological conditions (Lehmann et al., 1987; Wackerman et al., 1993; Strik and Lehmann, 1993; Strik et al., 1995; Kinoshita et al., 1995). In evoked potential studies the segmentation procedure has repeatedly been applied to define sequential steps of information processing after stimulation. Examples of such applications are the demonstration of differences in functional microstate configuration, when processing different types of visual stimuli (Michel et al., 1992, 1999a), when perform- ing a mental rotation task of increasing complexity (Pegna et al., 1997), when processing words with a visual and abstract meaning (Koenig et al., 1998), when judging semantical or phonological relations of words (Khateb et al., 1999), and when performing visuo-motor tasks (Thut et al., 1999, 2000).

Besides identifying stable segments during the different phases of seizure development, localization of the brain sources corresponding to the different segments is of interest. Pegna et al. (1997) used a linear inverse solution algorithm to perform source localization on the map corresponding to the segment of interest in the mental rotation task. The same method was used by Thut et al. (1999) to identify the site of interhemispheric transfer, and by Khateb et al. (1999) to determine which areas were active in the semantic and phonological processing.

In the present study we wanted to investigate (1) whether space-oriented temporal segmentation of epileptic seizures would be possible by applying the concept of microstates as proposed by Lehmann (i.e. periods of relative stationary EEG activity with rapid transitions), and (2) whether a linear inverse solution technique might be used to reconstruct the sources of the different microstates, hence giving an indication of the initiation and spread of the ictal activity. Preliminary results have been presented in a review article by Michel et al. (1999b).

\section{Patients and methods}

The EEG of 9 patients (6 male, 3 female, aged 8-44 years, mean 24 years) who had undergone a presurgical work-up due to pharmacoresistant epileptic seizures was analyzed. All patients had complex partial seizures, in two cases with secondary generalization. They had been the subject of invasive recordings with subdural grid, strip or depth electrodes, and the ictal onset zone had been identified. Ictal onset was subtemporal in two cases, posterior temporal in one case, frontal in 3 cases, central in one case, and parietal in one case. The last patient had two separate ictal onset zones, left temporal and left frontal, the former being the most frequent one. Clinical information on the patients is given in Table 1 . Seven of the 9 patients were operated on with resection of the ictal onset zone. Five of these were seizure-free 15-40 months (mean 24 months) after surgery, one had shown a few suspect seizures 36 months postoperatively, and one had experienced $75 \%$ seizure reduction 12 months after surgery. In one case (patient 5) repeated subpial transections were performed with some improvement, and in the final case (patient 3) surgery was denied due to the proximity of the ictal onset zone to eloquent cortex.

In each patient, between two and 16 (mean 6) seizures were selected from a preoperative ictal recording with surface electrodes. The number of recording electrodes was 26 in 6 cases and 29 in 3 cases, and the sampling rate was 256 in 3 cases and 128 in 6 cases. In 7 cases the data set 
Table 1

Results from neuroimaging investigations, ictal onset zone, performed surgery and postoperative outcome of all patients ${ }^{\mathrm{a}}$

\begin{tabular}{|c|c|c|c|c|c|c|c|}
\hline Patient & Gender & $\begin{array}{l}\text { Age } \\
\text { (years) }\end{array}$ & MR & $\begin{array}{l}\text { SPECT interictal } \\
\text { (Ceretec) }\end{array}$ & Ictal onset & Surgery & Outcome \\
\hline 1 & $\mathrm{~F}$ & 44 & Wide $\mathrm{T}$ horn $\mathrm{Lt}$ & Lt FT & Lt subT & Lt T & 0 seizures 15 months \\
\hline 2 & M & 28 & Normal & Rt T* & Lt subT & Lt T & 0 seizures 24 months \\
\hline 3 & M & 32 & Arachnoidal cyst Lt TO & $\mathrm{Lt} F+\mathrm{T}$ & Lt post $\mathrm{T}$ & No surgery & \\
\hline 4 & M & 22 & Normal & $\mathrm{Lt}+\mathrm{Rt} \mathrm{F}+$ other & Rt lat post $F$ & Rt lat post $\mathrm{F}$ & 0 seizures 40 months \\
\hline 5 & M & 8 & Normal & Rt C & Rt sensorimotor area & $* *$ & Some improvement \\
\hline 6 & $\mathrm{~F}$ & 18 & Wide sulci Rt P & Rt TP & Rt TP & Rt TP & $\begin{array}{l}\text { Few suspect seizures } \\
3 \text { years }\end{array}$ \\
\hline 7 & M & 25 & Normal & Lt F (ictal) & Lt dorsolateral $\mathrm{F}+\mathrm{FC}$ & Lt F & 0 seizures 24 months \\
\hline 8 & M & 24 & Hypotroph Lt F, large Lt T lobe & Lt T (ictal) & $\mathrm{Lt} \mathrm{T}+\mathrm{Lt}$ lat bas F & $\mathrm{Lt} \mathrm{T}+\mathrm{F}$ & 0 seizures 15 months \\
\hline 9 & $\mathrm{~F}$ & 18 & Normal & Lt F + other & Lt F parasagittal & Lt F & $\begin{array}{l}75 \% \text { seizure } \\
\text { reduction } 12 \text { months }\end{array}$ \\
\hline
\end{tabular}

${ }^{\text {a }}$ T, temporal; F, frontal; C, central; O, occipital; P, parietal; Lt, left; Rt, right; lat, lateral; post, posterior; bas, basal. *Lomazenil: left temporal; **multiple subpial transections.

included a time interval from $16 \mathrm{~s}$ before to $48 \mathrm{~s}$ after the visually estimated seizure onset. In two patients with very short seizures (5-6 s duration) shorter time intervals were chosen: $8 \mathrm{~s}$ before to $8 \mathrm{~s}$ after onset in one case (patient 9), and $6 \mathrm{~s}$ before to $6 \mathrm{~s}$ after onset in the other (patient 5).

For details about the program used for segmentation/ fitting see Pascual-Marqui et al. (1995). In short, the program operates as follows. Given the requested number of clusters $n$, the program defines the $n$ most prominent surface voltage distributions in a data set using a $k$ means spatial cluster analysis (segmentation), and determines for each timepoint which of these $n$ voltage distributions explains the data best (fitting). Similar voltage distributions of opposite polarities are assigned to the same cluster. The optimal number of clusters $n$ can be determined for each data set through a cross-validation criterion.

In each patient the following analyses were performed. For each seizure, peaks in the global field power (GFP, the spatial standard deviation) were identified, and a new data matrix was constructed consisting of all such GFP peaks in order of appearance (from here on referred to as the peak matrix). It has previously been demonstrated (Lehmann et al., 1987; Wackerman et al., 1993) that surface EEG maps tend to be stable around GFP maxima, and that confining the analysis to these maxima provides a means for decreasing the amount of data without loosing significant information. The segmentation/fitting program was then applied to the peak matrices of all seizures, giving the over all seizures optimal number of clusters $n$ determined through a crossvalidation criterion, and the voltage maps corresponding to each of the $n$ clusters. In a final step it was determined during which periods of each seizure these predefined maps occurred.

The output of the entire analysis was thus a number of segment maps best explaining the activity in all seizures of a patient, and their temporal occurrence in the peak matrix of each seizure.
A comprehensive presentation of all segment maps in all patients, and their variation over time in each seizure, would be too space consuming. For this reason the results were quantified as follows. Each raw data file of each patient was divided into 4 equal time periods. The duration of each subperiod was 3-4 s for the two cases with short seizures, and $16 \mathrm{~s}$ in the other cases. The subperiods before the visually estimated seizure onset were labeled pIct (for preictal), and those after seizure onset Ict (for ictal) with numbering throughout. Then the percentage time $t \%$ occupied by each of the $n$ different segment maps was determined based on the segmentation/fitting results. In each patient and for each subperiod an overall seizure average of $t \%$ for each segment map was calculated. By comparing this averaged $t \%$ for the different subperiods, a measure of the temporal development of the different segment maps in a patient was obtained (Table 2). In other words, the data sets were divided into shorter time periods, and an overall seizure average of the time occupied by each segment map during the different time periods was calculated.

Finally, after interpolating the EEG of all patients to the same standard 21 electrode setup, the different segment maps were subjected to a linear inverse solution algorithm: column weighted minimum norm (WMN) (Grave de Peralta Menendez and Gonzalez Andino, 1998). We chose the RWMN algorithm because it avoids the undesirable tendency of the unweighted minimum norm solution to reach maxima only at the borders of the solution space. This algorithm has shown a performance comparable or better than more complex solutions in the analysis of epileptic data and evoked potentials (Michel et al., 1999b). The solutions were calculated using a method recently developed at our lab (Spinelli et al., 2000) which enables the solution points to be constrained according to the anatomy of a realistic brain. Since MRI data could not be obtained from all the individual patients the reconstruction was performed in a generic brain. 
Table 2

The results of the segmentation in each patient ${ }^{\mathrm{a}}$

\begin{tabular}{llllllllll}
\hline \multicolumn{3}{l}{ Map } & & & & & & & \\
\cline { 2 - 7 } & 1 & 2 & 3 & 4 & 5 & 6 & 7 \\
\hline
\end{tabular}

\begin{tabular}{lcccrrrrr}
\hline $\begin{array}{l}\text { Patient 1 }(5 ; 26 ; 256) \\
\text { Subperiod } \\
\text { pIct1 }\end{array}$ & 38 & $\mathbf{8}$ & 9 & 0 & 7 & 9 & 30 \\
\hline Ict1 & 7 & $\mathbf{5 3}$ & 17 & 0 & 7 & 7 & 8 \\
Ict2 & 2 & $\mathbf{4 4}$ & 13 & 6 & 19 & 14 & 1 \\
Ict3 & 6 & $\mathbf{2 0}$ & 28 & 23 & 7 & 9 & 6
\end{tabular}

Patient 2 (4; 26; 256)

MapA MapB

Subperiod

\begin{tabular}{lrrrrrr} 
pIct1 & 48 & $\mathbf{1 2}$ & $\mathbf{4}$ & 10 & 10 & 16 \\
\hline Ict1 & 11 & $\mathbf{5 4}$ & $\mathbf{8}$ & 20 & 3 & 4 \\
Ict2 & 2 & $\mathbf{2 0}$ & $\mathbf{5 7}$ & 16 & 0 & 5 \\
Ict3 & 3 & $\mathbf{6}$ & $\mathbf{6 1}$ & 2 & 17 & 12 \\
Patient 3 $(2 ; 26 ; 128)$ & & MapA & MapB & & &
\end{tabular}

\begin{tabular}{lrrrrrr}
\hline $\begin{array}{l}\text { Subperiod } \\
\text { pIct1 }\end{array}$ & 49 & $\mathbf{8}$ & $\mathbf{2 4}$ & 18 & 1 & 0 \\
\hline Ict1 & 8 & $\mathbf{4 6}$ & $\mathbf{3 1}$ & 12 & 0 & 4 \\
Ict2 & 0 & $\mathbf{3}$ & $\mathbf{5 2}$ & 2 & 13 & 30 \\
Ict3 & 11 & $\mathbf{9}$ & $\mathbf{5}$ & 30 & 28 & 16 \\
& & & & & &
\end{tabular}

\begin{tabular}{lrrrrr}
\hline $\begin{array}{l}\text { Subperiod } \\
\text { pIct1 }\end{array}$ & 38 & $\mathbf{3 1}$ & 20 & 3 & 8 \\
\hline Ict1 & 0 & $\mathbf{6 8}$ & 11 & 20 & 2 \\
Ict2 & 14 & $\mathbf{3 4}$ & 38 & 9 & 4 \\
Ict3 & 17 & $\mathbf{1 8}$ & 30 & 28 & 6 \\
& & & & \\
Patient 5 $(13 ; 26 ;$ 128) & & &
\end{tabular}

\begin{tabular}{lccc}
\hline $\begin{array}{l}\text { Subperiod } \\
\text { pIct1 }\end{array}$ & & & \\
pIct2 & 48 & 41 & $\mathbf{1 0}$ \\
\hline Ict1 & 39 & 48 & $\mathbf{1 3}$ \\
Ict2 & 24 & 10 & $\mathbf{6 6}$ \\
& 6 & 10 & $\mathbf{8 3}$ \\
Patient 6 $(3 ; 26 ; 256)$ & MapA & MapB &
\end{tabular}

\begin{tabular}{lllll}
\hline $\begin{array}{l}\text { Subperiod } \\
\text { pIct1 }\end{array}$ & $\mathbf{6 1}$ & $\mathbf{2 1}$ & 0 & 18 \\
\hline Ict1 & $\mathbf{3 8}$ & $\mathbf{2 6}$ & 7 & 29 \\
Ict2 & $\mathbf{1 4}$ & $\mathbf{5 7}$ & 16 & 13 \\
Ict3 & $\mathbf{3 5}$ & $\mathbf{3 0}$ & 25 & 11 \\
& & & & \\
Patient $7(4 ; 29 ; 128)$ & MapA & MapB & MapC
\end{tabular}

\begin{tabular}{lrrrrrrr}
\hline $\begin{array}{l}\text { Subperiod } \\
\text { pIct1 }\end{array}$ & $\mathbf{6 9}$ & $\mathbf{7}$ & $\mathbf{4}$ & 0 & 1 & 18 & 2 \\
\hline Ict1 & $\mathbf{4 0}$ & $\mathbf{2 1}$ & $\mathbf{1 0}$ & 0 & 6 & 15 & 9 \\
Ict2 & $\mathbf{4}$ & $\mathbf{6 0}$ & $\mathbf{7}$ & 3 & 3 & 12 & 10 \\
Ict3 & $\mathbf{4}$ & $\mathbf{1 2}$ & $\mathbf{2 4}$ & 21 & 14 & 11 & 14
\end{tabular}

Table 2 (continued)

\begin{tabular}{|c|c|c|c|c|c|c|c|}
\hline & \multicolumn{7}{|c|}{ Map } \\
\hline & 1 & 2 & 3 & 4 & 5 & 6 & 7 \\
\hline Patient $8(3 ; 29 ; 128)$ & & MapA & MapB & MapC & & & \\
\hline $\begin{array}{l}\text { Subperiod } \\
\text { pIct } 1\end{array}$ & 34 & 12 & 5 & 6 & 13 & 15 & 16 \\
\hline Ict 1 & 13 & 43 & 25 & $\mathbf{0}$ & 3 & 8 & 8 \\
\hline Ict2 & 13 & 10 & 12 & 27 & 10 & 14 & 13 \\
\hline Ict 3 & 20 & 7 & 29 & 16 & 9 & 13 & 6 \\
\hline Patient $9(16 ; 29 ; 128)$ & & MapA & MapB & MapC & & & \\
\hline Subperiod & & & & & & & \\
\hline pIct 1 & 46 & 22 & 7 & 8 & 12 & 6 & \\
\hline pIct2 & 41 & 21 & 11 & 12 & 12 & 3 & \\
\hline Ict 1 & 9 & 39 & 21 & 22 & 8 & 1 & \\
\hline Ict2 & 8 & 11 & 20 & 38 & 19 & 2 & \\
\hline $\begin{array}{l}\text { a Numbers in the tabl } \\
\text { incidence of the differe } \\
\text { Numbers within parenth } \\
\text { of extracranial recording } \\
\text { Ict } 1-3 \text { represent differen } \\
\text { tant maps during the sei } \\
\text { these maps are shown in } \\
\text { onset. }\end{array}$ & $\begin{array}{l}\text { gese } \\
\text { t pha } \\
\text { t pure }\end{array}$ & $\begin{array}{l}\text { he over } \\
\text { e maps } \\
\text { cate the } \\
\text { les, and } \\
\text { of seizur } \\
\text { given ir } \\
\text { Thick lir }\end{array}$ & $\begin{array}{l}\text { all seizu } \\
\text { in the di } \\
\text { number } \\
\text { the samp } \\
\text { e develop } \\
\text { bold ty } \\
\text { es repres }\end{array}$ & $\begin{array}{l}\text { re avera } \\
\text { fferent ti } \\
\text { of seizur } \\
\text { ling rate } \\
\text { ment (se } \\
\text { pe. Inver } \\
\text { sent the }\end{array}$ & $\begin{array}{l}\text { ged } \\
\text { me s } \\
\text { es, th } \\
\text { pIct } \\
\text { e tex } \\
\text { se so } \\
\text { ime }\end{array}$ & $\begin{array}{l}\text { perce } \\
\text { eque } \\
\text { te nu } \\
t 1(-2) \\
\text { t). In } \\
\text { lutio }\end{array}$ & $\begin{array}{l}\text { entual } \\
\text { ences. } \\
\text { imber } \\
\text { mpor and } \\
\text { npor- } \\
\text { ns of } \\
\text { izure }\end{array}$ \\
\hline
\end{tabular}

\section{Results}

The results of the segmentation in each patient, displayed as the percentage incidence of all segment maps over the different subperiods, are shown in Table 2. The results from the source reconstructions of the most important segment maps are shown in Fig. 1.

In both patient 1 and patient 2 , the segment map dominating during the earliest ictal subperiod Ict1 (labeled mapA in both cases) has a left temporal source (Fig. 1 and Table 2). These findings are consistent with a left subtemporal seizure onset seen in the subdural recordings. In the latter patient a second segment (mapB) evolves during Ict2-3. The source reconstruction result of this map is only slightly different from that of mapA (for details see Fig. 2). The patients are seizure-free 15 and 24 months after left temporal lobe resection.

In patient 3 , the map dominating during Ict1 (mapA) has a source distribution with a left occipital maximum. MapB, which is second dominant during Ict1 and dominant during Ict2, has a left temporal maximum (Fig. 1 and Table 2). The intracranial recording shows seizure onset posterior temporal left, which does not exactly correspond to the location of any of the segment maps, although both are relatively close. Due to the proximity of the ictal onset zone to eloquent cortex, surgery was denied in this patient.

In patient 4 , the map dominating during Ict1 (mapA) has a right frontal source maximum (Fig. 1 and Table 2). 

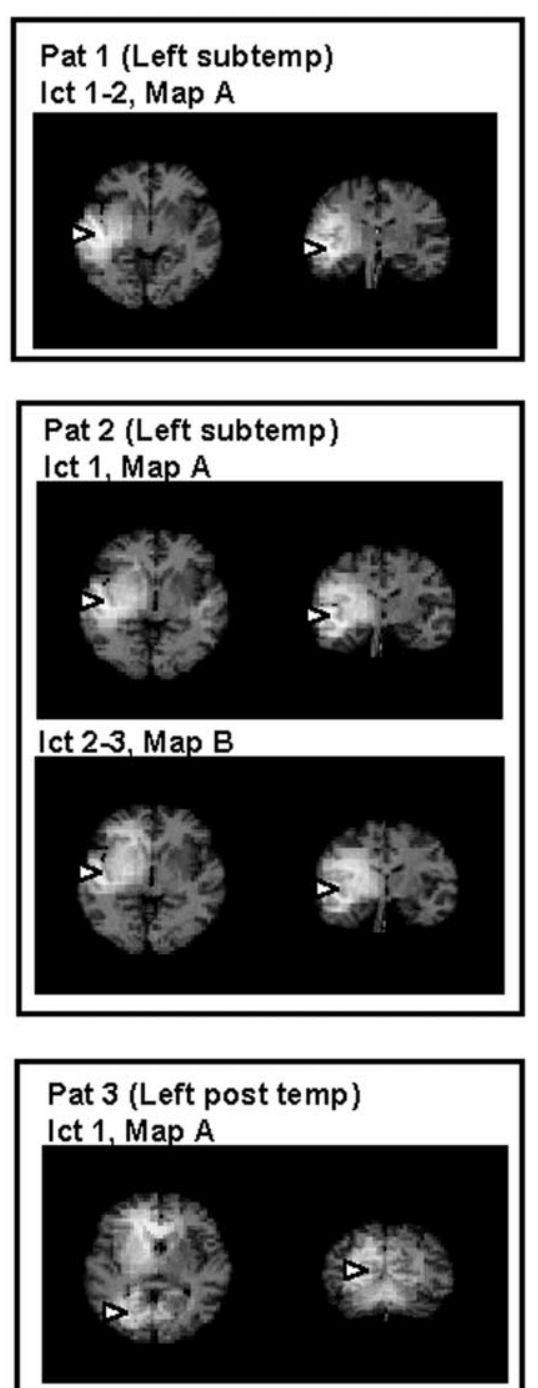

Ict 2, Map B
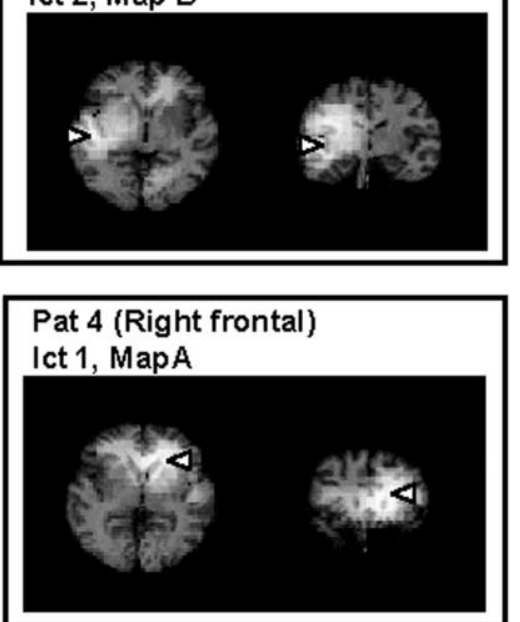
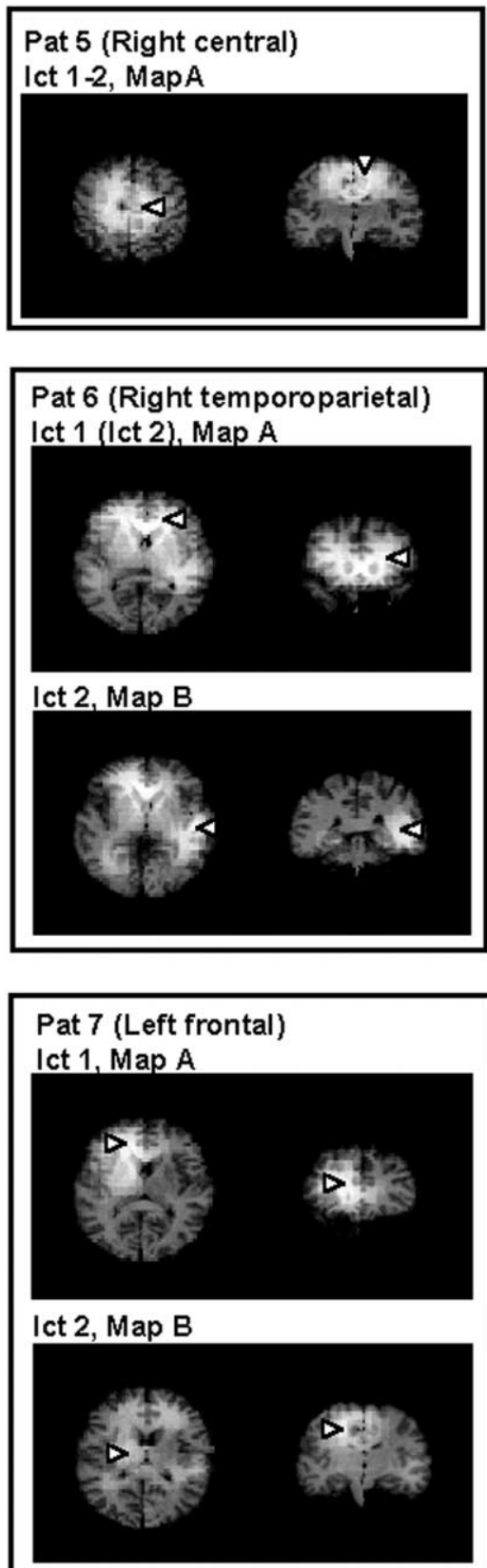

Ict 3, Map C

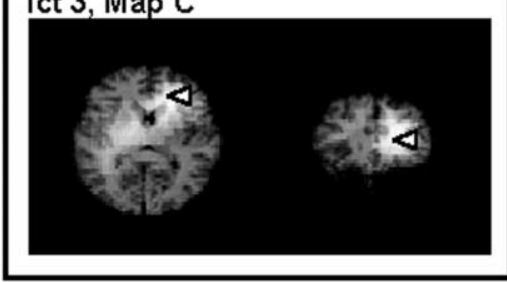

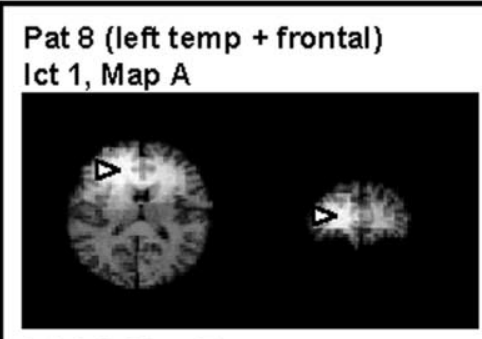

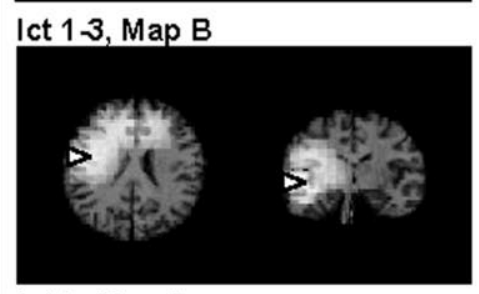

Ict 2, Map C
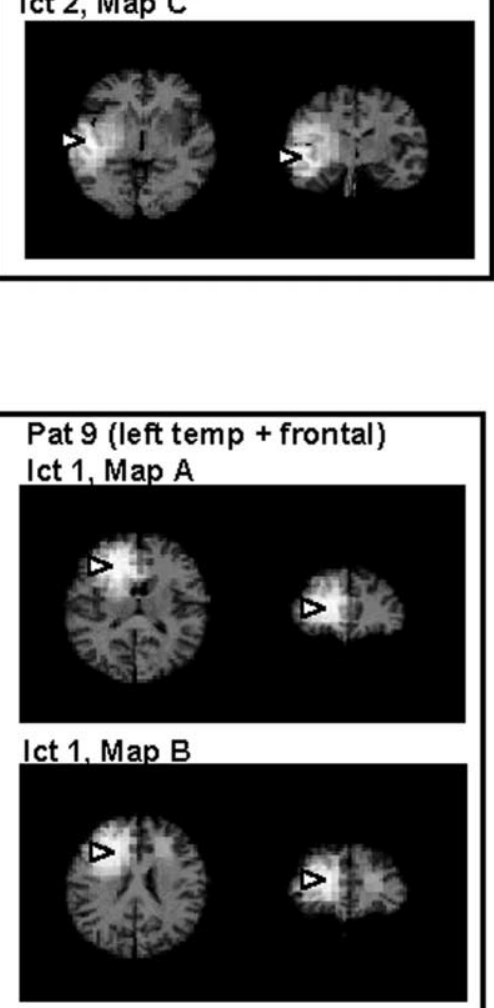

Ict 1+2, Map C

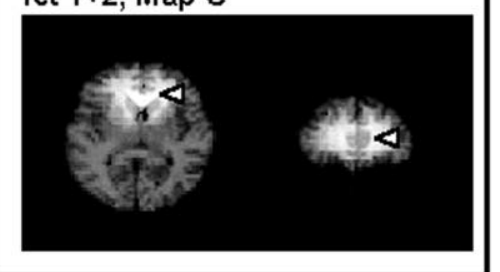

Fig. 1. Source reconstruction results of the most important maps in all patients. Text within parentheses indicates the approximate ictal onset area. Text below indicates during which of the time sequences Ict1-3 the source is most active and the label of the corresponding source reconstruction in Table 2. The slice in the horizontal and coronal planes containing the point with the highest activity is displayed, and the arrow indicates the location of this point. 


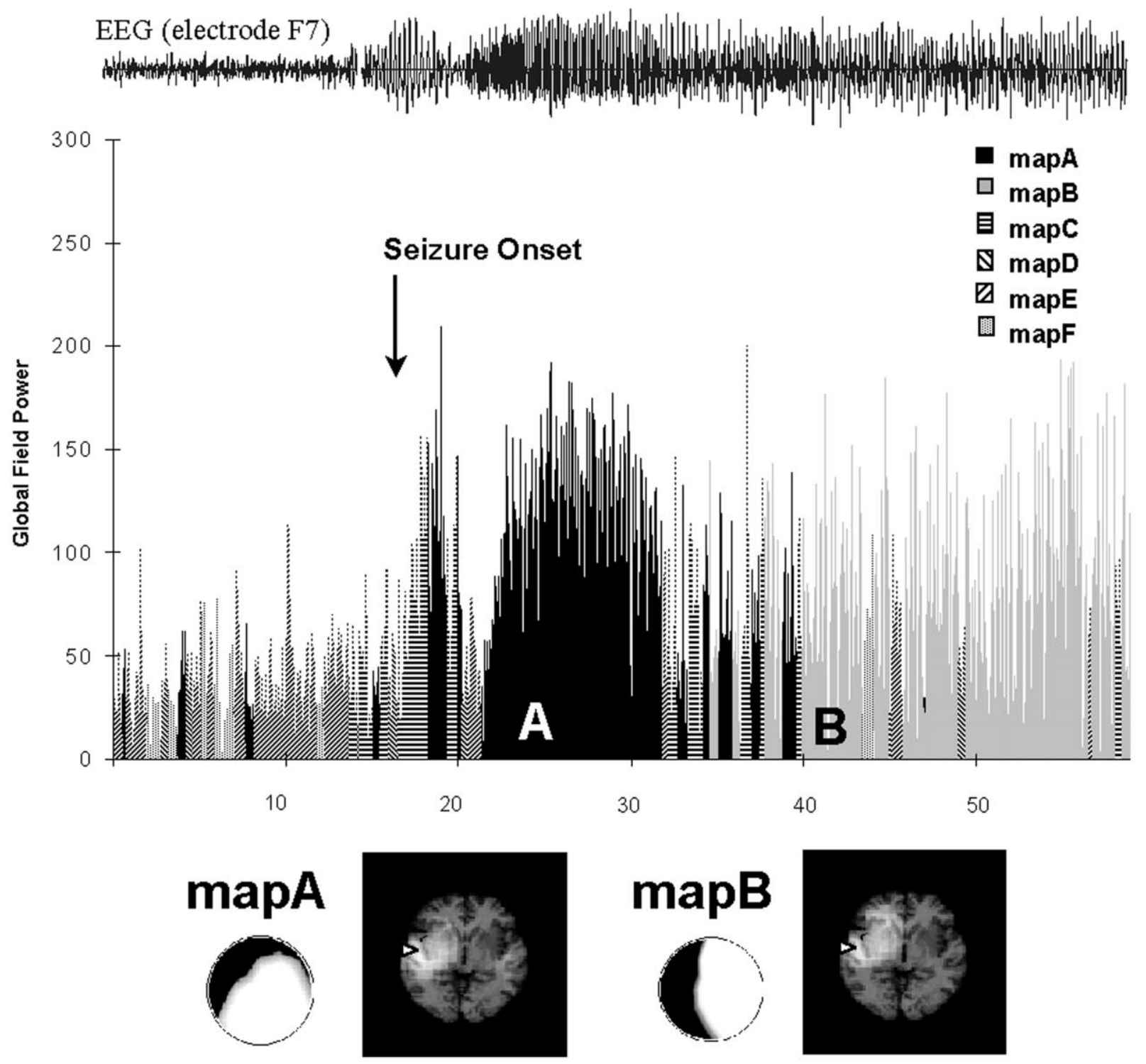

Fig. 2. Surface EEG (electrode F7), segmentation results, surface maps, and source reconstruction results of patient 2. For each timepoint in the peak matrix (GFP peaks, see text) the GFP and the assigned map are displayed. Before seizure onset GFP is low, and mapE is dominating. Around the visually estimated seizure onset there is a short increase in GFP, the peak of which is assigned to mapA (black). Directly after this, another longer period of high GFP is seen, and throughout this period ( $5 \mathrm{~s}$ ) the surface maps of all timepoints are assigned to mapA (black). Later in seizure development GFP remains at a high level, and most of the timepoints are assigned to mapB (gray), although with short interruptions by other maps. Although source reconstructions show left temporal seizure location for the two dominating ictal surface maps (mapA and mapB), different map configurations and a certain change of the source reconstruction results indicate somewhat different ictal activity distributions for the two segments. The different source reconstruction results for the two maps may have their counterpart in an intracranially verified ictal spread from the left subtemporal area to major parts of the left temporal lobe, although the source reconstruction rather indicates spread to the left frontal region. The patient is seizure-free 24 months after left temporal lobe resection.

The intracranial recordings show seizure onset frontal right, in good correspondence with the maximum of mapA. The patient is seizure-free 40 months after frontal lobe resection.

In patient 5, a map with a right central maximum (mapA) is clearly dominating during Ict1-2 (Fig. 1 and Table 2). The location of this maximum corresponds well to the location of intracranial seizure onset (right sensorimotor area). The patient has undergone multiple subpial transections of this area with some improvement of the seizure situation.

In patient 6 , the map dominating before seizure onset
(mapA with a bifrontal source maximum, but with a right lateral component) is still most prominent in the early ictal period (Fig. 1 and Table 2). However, mapB (right temporoparietal), and a map with a diffuse right frontal source (data not shown) also occupy a significant part of this time period. During Ict2 mapB becomes clearly dominant. The source location of mapB corresponds well to the location of intracranial seizure onset (right parietal). The patient has had a few suspect seizures 36 months after a right parietal resection. 
In patient 7, a left frontal activity (mapA) is dominating before seizure onset (pIct1) and early in seizure development (Ict1) (Fig. 1 and Table 2). The intracranial seizure onset (left frontal) is consistent with the distribution of this source, although the total dominance of the corresponding map also before seizure onset is difficult to explain. Two additional maps (mapB and mapC), with a vertex and a right frontal maximum, dominate during Ict 2 and Ict3 (Fig. 1 and Table 2). There is no direct intracranial counterpart to the maxima of these maps. The patient is seizure-free 24 months after left frontal resection.

In patient 8, mapA (bifrontal with left predominance) dominates during Ict1, but mapB (left temporal maximum with a bifrontal left dominant component) also occupies a significant part of this time period (Fig. 1 and Table 2). During Ict2 a third map (mapC) with a localized left temporal source becomes dominant (Fig. 1 and Table 2). In this patient two ictal onset zones were encountered, the more frequent left temporal (corresponding to mapB and mapC), and the less frequent left lateral frontobasal (corresponding to the dominant mapA). The patient experienced more than $75 \%$ seizure reduction 12 months after a left temporal resection. Due to few but still handicapping seizures an additional resection of the left lateral frontal area was performed, and the patient is seizure-free 15 months after the last resection.

In patient 9, a left frontal activity (mapA) dominates during Ict1 (Fig. 1 and Table 2), but mapB (left frontal with slightly different source distribution) and mapC (frontocentral + right frontal) also occupy a significant part of the time (Fig. 1 and Table 2). During the second phase of the short seizures (Ict2) mapC is the most frequent. Intracranial recordings demonstrate seizure onset in the left frontal region. The patient has experienced more than $75 \%$ seizure reduction 24 months after left frontal resection.

In short, it was possible in 7 of the 9 cases to identify one segment map which was clearly dominant during the early phases of the seizures. In 6 of these cases (patients 1, 2, 4, 5, 7 , and 9) the source reconstruction of this map corresponded well to intracranial seizure onset. In the last of these 7 cases (patient 8 ) the most active map corresponded to one, and the second most active map to another ictal onset area. In one of the 9 cases (patient 6) the initial subperiod of the seizure contained several about equally active segment maps, one of which corresponded to the intracranial onset, but with the most active map having a different distribution. In this case the 'correct' map evolved to be most active during the subsequent time period. Finally, in patient 3, two about equally active segments both gave source reconstruction results in the vicinity of the intracranial onset zone, although none of them showed a perfect match.

\section{Discussion}

The main advantage of EEG compared to other methods for epileptic focus localization is its high temporal resolution. It is demonstrated by intracranial recordings that the distribution of activity in the brain may change within fractions of a second as the ictal activity propagates from one area to another. Even if the high temporal resolution of EEG gives excellent and unique possibilities to visualize these fast changes, it also entails problems concerning the interpretation of the fast transitions between the different surface voltage distributions. For spontaneous EEG the concept of microstates was introduced by Lehmann et al. (1987) as a means of quantifying these changes in EEG topography. The concept is based on the observation that surface voltage distributions of spontaneous EEG remain relatively stable over short time periods (called segments), and that fast changes occur between these periods of relative stationarity. It was suggested that these periods of relative stability, called microstates, would manifest different brain functional states. The rationale behind the present investigation was that the observed features of ictal activity would make adaptive segmentation, as proposed by Lehmann, a useful method for quantification of seizure onset and development.

When analyzing epileptic seizures several tens of seconds of EEG have to be studied, and if the segmentation were to be performed on continuous EEG over such long time periods, very large amounts of data would have to be analyzed. For this reason some kind of primary data reduction is necessary. It has previously been demonstrated (Lehmann et al., 1987; Wackerman et al., 1993) that surface EEG maps tend to be stable around GFP maxima, and that confining the analysis to these maxima provides a means for decreasing the amount of data without loosing significant information. Hence, by confining to GFP peaks, the amount of data to be analyzed in this investigation could be reduced by more than $90 \%$.

To fully visualize the usefulness of the method one has to consider the more comprehensive results, here exemplified by one seizure in each of two patients (Figs. 2 and 3). For the sake of brevity, however, we found it necessary in the remaining cases to present the results in a quite simplified way. The seizures were thus divided into subperiods of between 3 and $16 \mathrm{~s}$ duration, and the results presented were obtained by making across-seizure averages of the results during these subperiods. With this approach the risk of loosing relevant information is obvious. Many different events may occur during $16 \mathrm{~s}$, and inter-seizure variability may obscure the results when the results of several seizures are averaged. In order to diminish this problem, not only the most dominant segment, but rather all segments occupying a certain amount of the time in the early ictal period (arbitrarily set to $20 \%$ of the total time) are described in Section 3.

In 7 out of 9 cases a source configuration consistent with intracranial seizure onset was dominating during the initial phase of the seizures, and in one case (patient 6) such a configuration became dominant in the subsequent phase. Only in one case (patient 3, see below) was there a certain 

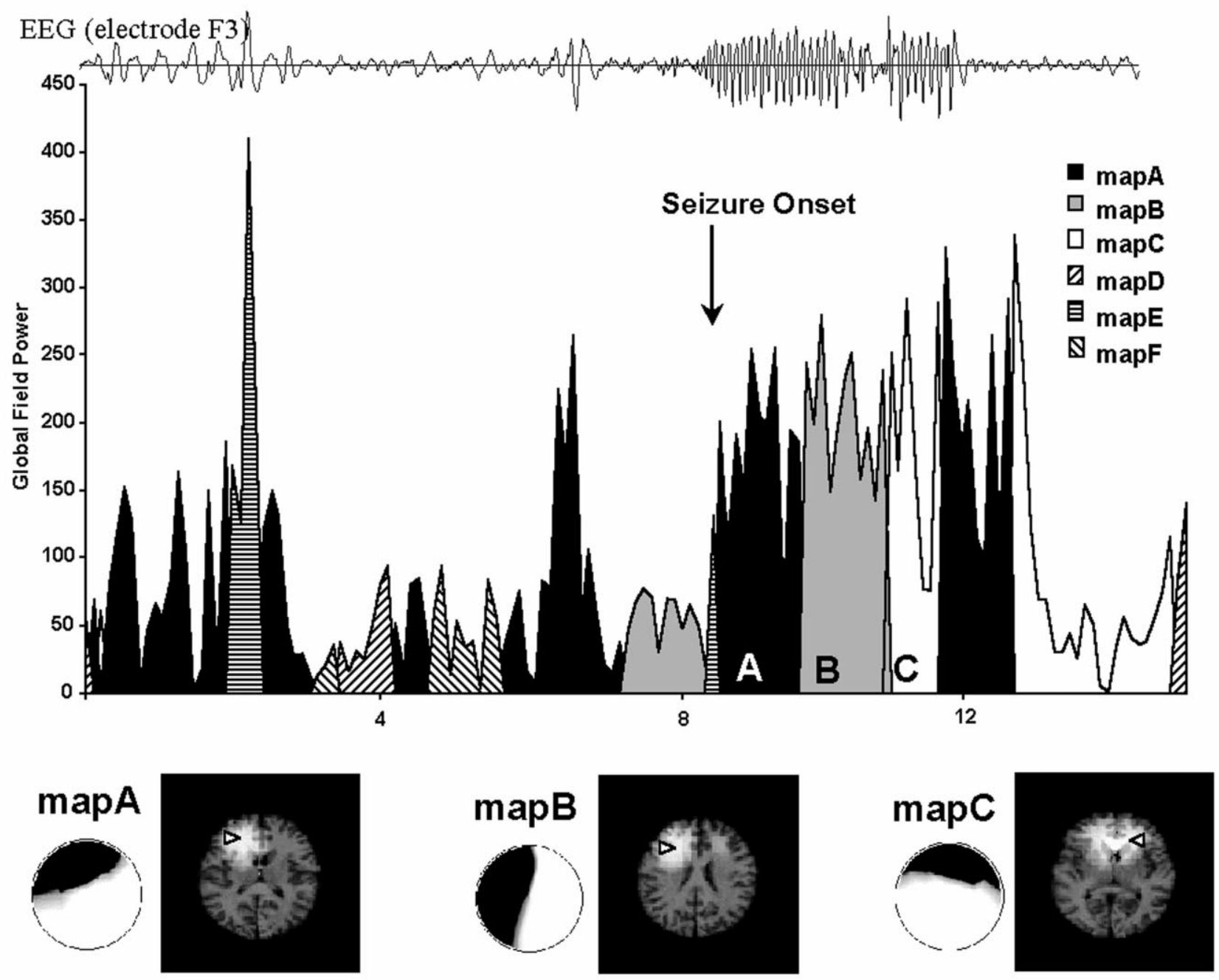

Fig. 3. Surface EEG (electrode F3), segmentation results, surface maps, and source reconstruction results of patient 9. For each timepoint in the peak matrix (GFP peaks, see text) the GFP and the assigned map are displayed. Before seizure onset GFP is low, except for a few peaks corresponding to interictal epileptiform potentials. Around the visually estimated seizure onset there is a distinct increase in GFP, and during the first $1-2 \mathrm{~s}$ after seizure onset all timepoints are assigned to mapA (black). During the next 1-2 s of the seizure all timepoints are mapB (gray) and, with a short interruption by mapA, the rest of the 7-8 s long seizures are assigned to mapC (white). Source reconstructions show a left frontal location of mapA and mapB, but with a weak right frontal activation for the latter. For mapC the source distribution is bifrontal with right predominance. Intracranial recordings show left frontal seizure onset. The apparent ictal spread from left to right frontal areas in this patient is to some extent supported by findings in spike triggered fMRI (Seeck et al., 1998), although this can not be definitely confirmed. The patient has more than $75 \%$ seizure reduction 24 months after left frontal lobe resection.

mismatch between the source reconstructions and ictal onset zones. Consequently, there is a high concordance between the segmentation/source reconstruction results and seizure onset. In the later time periods the results were more difficult to interpret, probably to some extent due to an increasing inter-seizure variability for the later parts of the seizures. Apart from the two cases mentioned above, another two patients (patients 8 and 9) showed rather complex segmentation results, with several segments active during the early phases of the seizures (Table 2). Three of these 4 patients have been operated on, but only one is completely seizurefree, although the number of seizures in the other two have decreased dramatically (a few suspect seizures in patient 6 , and more than $75 \%$ seizure reduction in patient 9 ). Four of the 5 patients who had one clearly dominating segment have undergone resective surgery and all are seizure-free. Consequently, apart from the localizing information, the segmentation results might also be used to get an impression about the complexity of the seizures.

In one of the cases (patient 3) there was a certain mismatch between the source reconstructions and the ictal onset. In this patient, mapA (left occipital) is dominating during Ict1, while mapB (left temporal) is the most active during Ict2. Neither of these two maps correspond well with the intracranially confirmed ictal onset (left posterior temporal), although both are relatively close. The MRI in this patient shows an arachnoidal cyst in the left temporooccipital region, and a factor which might induce a mislo- 
calization in this case is that the reconstruction matrix (obtained from a normal brain) is quite different from the patient's brain in the area around the cyst. It is thus possible that the two maps represent sequential activation of the posterior and anterior border of this lesion. In order to confirm this theory, however, reconstruction in the patient's own brain together with co-registration of different modalities would be necessary.

In addition to the ictal onset maps, a sequence of additional maps was obtained in 6 of the 9 cases. In some of these cases there are some indications that the sequence of maps might reflect ictal propagation (Figs. 2 and 3). However, both the number of patients with similar onset and the number of seizures in each patient are rather small, and the evidence that the sequence of maps actually reflects ictal propagation is rather weak. We are currently in the process of making a large database with seizures from all our patients, and once this database is finished we intend to perform a more comprehensive study using seizure segmentation to assess ictal propagation in a larger group of patients.

In this investigation the recordings were made in a clinical setting that was not optimal for source reconstructions (relatively few electrodes, standard electrode positions, etc.). One somewhat unexpected finding that might be accounted for by this fact is the rather dorsal source distributions obtained for the two temporal lobe cases (patients 1 and 2). A possible explanation is that the reconstruction matrix of the WMN algorithm does not extend below the T3-T4 plane, and that sources with their electrical maximum at or below this level may become distorted. Consequently, in order to increase the reconstruction accuracy of temporal lobe sources, an extended reconstruction matrix including lower temporal areas should be applied. Another imperative development which is currently being made is the use of exact electrode positions and the use of realistic head models for the inverse solutions (Michel et al., 1999b, Appendix; Fuchs et al., 1999).

One interesting approach would be to combine segmentation with frequency analysis. The segmentation might be used to define periods of relative stationarity in seizure development, thus avoiding the problem of determining time borders for the frequency analysis. In this way the frequency spectrum of the ictal activity within a segment could be obtained, and a subsequent FFT dipole approximation could be used to localize the sources corresponding to peak frequencies (Lantz et al., 1999; Blanke et al., 2000).

We conclude that segmentation of ictal EEG, with subsequent 3-dimensional source reconstruction, is a useful method to non-invasively determine the seizure onset and possibly also the spread of epileptiform activity in patients with epileptic seizures. Further development will include the combination of this technique, having an inherently favorable time resolution, with more spatially, but less temporally, accurate functional imaging techniques such as fMRI, PET, or SPECT (Michel et al., 1999b).

\section{Acknowledgements}

This project was supported by Grant K97-14X-84-33C from the Swedish Medical Research Council, by the Medical Faculty, University of Lund, and by the Swiss National Science Foundation grants 31-57112.99 and 31-52933.97.

\section{References}

Alarcon G, Guy CN, Binnie CD, Walker SR, Elwes RDC, Polkey CE. Intracerebral propagation of interictal activity in partial epilepsy: implications for source localisation. J Neurol Neurosurg Psychiatry 1994;57:435-449.

Assaf BA, Ebersole JS. Continuous source imaging of scalp ictal rhythms in temporal lobe epilepsy. Epilepsia 1997;38:1114-1123.

Blanke O, Lantz G, Seeck M, Spinelli L, Grave de Peralta R, Thut G, Landis T, Michel CM. Temporal and spatial determination of EEGseizure onset in the frequency domain. Clin Neurophysiol 2000;111:763-772.

Boon P, D’Havé M, Adam C, Vonck K, Baulac M, Vandekerckhove T, De Reuck J. Dipole modeling in epilepsy surgery candidates. Epilepsia 1996;38:208-218.

Brekelmans GJF, van Emde Boas W, Velis DN, van Huffelen AC, Debets RMC, van Veelen CWM. Mesial temporal versus neocortical temporal lobe seizures: demonstration of different electroencephalographic spreading patterns by combined use of subdural and intracerebral electrodes. J Epilepsy 1995;8:309-320.

Bullmore ET, Brammer MJ, Bourlon P, Alarcon G, Polkey CE, Elwes R, Binnie CD. Fractal analysis of electroencephalographic signals intracerebrally recorded during 35 epileptic seizures: evaluation of a new method for synoptic visualisation of ictal events. Electroenceph clin Neurophysiol 1994;91:337-345.

Ebersole JS, Pacia SV. Localization of temporal lobe foci by ictal EEG patterns. Epilepsia 1996;37:386-399.

Franaszczuk PJ, Bergey GK, Kaminski MJ. Analysis of mesial temporal seizure onset and propagation using the directed transfer function method. Electroenceph clin Neurophysiol 1994;91:413-427.

Franaszczuk PJ, Bergey GK, Durka PJ, Eisenberg HM. Time-frequency analysis using the matching pursuit algorithm applied to seizures originating from the mesial temporal lobe. Electroenceph clin Neurophysiol 1998;106:513-521.

Fuchs M, Wagner M, Köhler T, Wischmann H-A. Linear and nonlinear current density reconstructions. J Clin Neurophysiol 1999;16:267-295.

Gath I, Lehmann D, Bar-On E. Fuzzy clustering of EEG signals and vigilance performance. Int J Neurosci 1983;20:303-312.

Gath I, Michaeli A, Feuerstein C. A model for dual channel segmentation of the EEG signal. Biol Cybern 1991;64:225-230.

Gath I, Feuerstein C, Pham DT, Rondouin G. On the tracking of rapid dynamic changes in seizure EEG. IEEE Trans Biomed Eng 1992;39:952-958.

Gotman J, Levtova V, Olivier A. Frequency of the electroencephalographic discharge in seizures of focal and widespread onset in intracerebral recordings. Epilepsia 1995;36:697-703.

Grave de Peralta Menendez R, Gonzalez Andino SL. A critical analysis of linear inverse solutions. IEEE Trans Biomed Eng 1998;45:440-448.

Kanemoto K, Kawasaki J, Kawai I. The lateralizing value of scalp ictal EEG patterns in temporal lobe epilepsy with unilateral hippocampal atrophy with special attention to the initial slow waves. J Epilepsy 1997; 10:225-231.

Khateb A, Annoni JM, Landis T, Pegna AJ, Custodi MC, Fonteneau E, Morand SM, Michel CM. Spatio-temporal analysis of electric brain activity during semantic and phonological word processing. Int $\mathrm{J}$ Psychophysiol 1999;32:215-231.

Kinoshita T, Strik WK, Michel CM, Yagyu T, Saito M, Lehmann D. Micro- 
state segmentation of spontaneous multichannel EEG map series under diazepam and sulpiride. Pharmacopsychiatry 1995;28:51-55.

Koenig T, Kochi K, Lehmann D. Event-related electric microstates of the brain differ between words with visual and abstract meaning. Electroenceph clin Neurophysiol 1998;106:535-546.

Lantz G, Michel CM, Seeck M, Blanke O, Landis T, Rosén I. Frequency domain EEG source localization of ictal epileptiform activity in patients with partial complex epilepsy of temporal lobe origin. Clin Neurophysiol 1999;110:176-184.

Lehmann D, Michel CM. Intracerebral dipole source localization for FFT power maps. Electroenceph clin Neurophysiol 1990;76:271-276.

Lehmann D, Ozaki H, Pal I. EEG alpha map series: brain micro-states by space-oriented adaptive segmentation. Electroenceph clin Neurophysiol 1987;67:271-288.

Lehnertz K, Elger CE. Spatio-temporal dynamics of the primary epileptogenic area in temporal lobe epilepsy characterized by neuronal complexity loss. Electroenceph clin Neurophysiol 1995;95:108-117.

Lopes da Silva FH, Mars NJI. Parametric methods in EEG analysis. In: Gevins AS, Rémond A, editors. Methods of analysis of brain electrical and magnetic signals, Handbook of electroencephalography and clinical neurophysiology, 1. Amsterdam: Elsevier, 1987. pp. 243-260.

Michael D, Houchin J. Automatic EEG analysis: a segmentation procedure based on the autocorrelation function. Electroenceph clin Neurophysiol 1979;46:232-235.

Michel CM, Henggeler B, Lehmann D. 42-channel potential map series to visual contrast and stereo stimuli: perceptual and cognitive eventrelated segments. Int J Psychophysiol 1992;12:133-145.

Michel CM, Seeck M, Landis T. Spatiotemporal dynamics of human cognition. News Physiol Sci 1999a;14:206-214.

Michel CM, Grave de Peralta R, Lantz G, Gonzalez Andino S, Spinelli S, Blanke O, Landis T, Seeck M. Spatio-temporal EEG analysis and distributed source estimation in presurgical epilepsy evaluation. J Clin Neurophysiol 1999b;16:225-238.

Osorio I, Frei MG, Wilkinson SB. Real-time automated detection and quantitative analysis of seizures and short term prediction of clinical onset. Epilepsia 1998;39:615-627.

Pacia SV, Ebersole JS. Intracranial EEG substrates of scalp ictal patterns from temporal lobe foci. Epilepsia 1997;38:642-654.

Pascual-Marqui RD, Michel CM, Lehmann D. Segmentation of brain electrical activity into microstates: model estimation and validation. IEEE Trans Biomed Eng 1995;42:658-665.

Pegna AJ, Khateb A, Spinelli L, Seeck M, Landis T, Michel CM. Unravelling the cerebral dynamics of mental imagery. Hum Brain Map 1997;5:410-421.

Pijn JP, van Neerven J, Noest A, Lopes da Silva FH. Chaos or noise in EEG signals; dependence on state and brain site. Electroenceph clin Neurophysiol 1991;79:371-381.
Risinger MW, Engel Jr J, Van Ness PC, Henry TR, Crandall PH. Ictal localization of temporal lobe seizures with scalp/sphenoidal recordings. Neurology 1989;39:1288-1293.

Sanderson AC, Segen J, Richey E. Hierarchical modelling of EEG signals. IEEE Trans Pattern Anal Machine Intel 1980;2:405-415.

Scherg M. Fundamentals of dipoles source analysis. In: Grandori F, Hoke M, Romani GL, editors. Auditory evoked electric fields and magnetic potentials, Advances in audiology, 6. Basel: Karger, 1990. pp. 40-96.

Seeck M, Lazeyras F, Michel CM, Blanke O, Gericke CA, Ives J, Delavelle J, Golay X, Haenggeli CA, de Tribolet N, Landis T. Non-invasive epileptic focus localization using EEG-triggered functional MRI and electromagnetic tomography. Electroenceph clin Neurophysiol 1998;106:508-512.

So N, Gloor P, Quesney LF, Jones-Gotman M, Olivier A, Andermann F. Depth electrode investigations in patients with bitemporal epileptiform abnormalities. Ann Neurol 1989;25:423-431.

Spencer SS, Guimaraes P, Katz A, Kim J, Spencer D. Morphological patterns of seizures recorded intracranially. Epilepsia 1992;33:537545.

Spinelli L, Gonzalez Andino S, Lantz G, Michel CM. Co-registration of distributed electromagnetic inverse solutions with magnetic resonance images using MRI-to-sphere transformation. Brain Topogr 2000;13:111.

Strik WK, Lehmann D. Data determined window size and space-oriented segmentation of spontaneous EEG map series. Electroenceph clin Neurophysiol 1993;87:169-174.

Strik WK, Dierks T, Becker T, Lehmann D. Larger topographical variance and decreased duration of brain electric microstates in depression. $\mathrm{J}$ Neural Transm Gen Sect 1995;99:213-222.

Thut G, Hauert C-A, Morand S, Seeck M, Landis T, Michel C. Evidence for interhemispheric motor-level transfer in a simple reaction time task: an EEG-study. Exp Brain Res 1999;128:256-261.

Thut G, Hauert C-A, Viviani P, Morand S, Spinelli L, Blanke O, Landis T, Michel C. Internally driven versus externally cued movement selection: a study on the timing of brain activity. Cogn Brain Res 2000;9:261269

Unser M, Aldroubi A. A review of wavelets in biomedical applications. Proc IEEE 1996;84:626-638.

Wackerman J, Lehmann D, Michel CM, Strik WK. Adaptive segmentation of spontaneous EEG map series into spatially defined microstates. Int J Psychophysiol 1993;14:269-283.

Wendling F, Badier JM, Chauvel P, Coatrieux JL. A method to quantify invariant information in depth-recorded epileptic seizures. Electroenceph clin Neurophysiol 1997;102:472-485.

Wu L, Gotman J. Segmentation and classification of EEG during epileptic seizures. Electroenceph clin Neurophysiol 1998;106:344-356. 